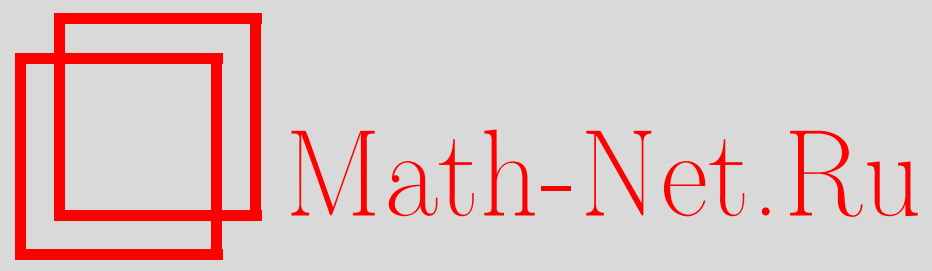

А. Н. Тимашёв, Об асимптотике вероятностей больших уклонений для отрицательного полиномиального распределения, Дискрет. матем., 2002, том 14, выпуск 1, 75-81

DOI: https://doi.org/10.4213/dm235

Использование Общероссийского математического портала Math-Net.Ru подразумевает, что вы прочитали и согласны с пользовательским соглашением http://www.mathnet.ru/rus/agreement

Параметры загрузки:

IP: 34.229 .45 .116

26 апреля 2023 г., 08:42:49

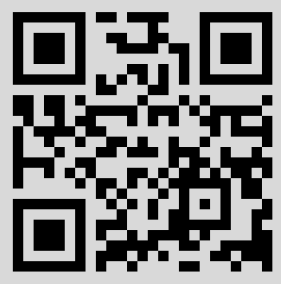




\title{
Об асимптотике вероятностей больших уклонений для отрицательного полиномиального распределения
}

\author{
(c) 2002 г. А. Н. Тимашёв
}

\begin{abstract}
Рассматривается полиномиальная схема испытаний, в которой имеется $N+1$ исходов $E_{0}, E_{1}, \ldots, E_{N}$ с вероятностями $p_{0}, p_{1}, \ldots, p_{N}$ соответственно. Предполагается, что испытания проводятся до $r$-й реализации исхода $E_{0}, r=1,2, \ldots$ Если $\eta_{j}(r)$ - число реализаций исхода $E_{j}$ в момент остановки испытаний, $j=1, \ldots, N$, и $\eta(r)=\left(\eta_{1}(r), \ldots, \eta_{N}(r)\right)$, то вектор $\eta(r)$ имеет отрицательное полиномиальное распределение. В предположении, что $N \in \mathbf{N}$ и положительные вероятности $p_{0}, p_{1}, \ldots, p_{N}$ фиксированы, и $r \rightarrow \infty$ и $k_{1}, \ldots, k_{N} \rightarrow \infty$ так, что параметры $\beta_{j}=k_{j} / r$ удовлетворяют неравенствам $\beta_{j} \geqslant \varepsilon$, где $\varepsilon$ - положительная постоянная, $j=1, \ldots, N$, и некоторым дополнительным условиям, найдены асимптотические оценки вероятностей больших уклонений $\mathbf{P}\left\{\eta_{j}(r) \leqslant k_{j}, j=1, \ldots, N\right\}$ и $\mathbf{P}\left\{\eta_{j}(r) \geqslant k_{j}, j=1, \ldots, N\right\}$. Для получения нужных асимптотических оценок используется многомерный метод перевала в варианте И. Гуда.
\end{abstract}

Рассмотрим полиномиальную схему испытаний, в которой имеется $N+1$ исходов $E_{0}, E_{1}, \ldots, E_{N}$. Всюду далее $N \in \mathbf{N}$ фиксировано. Пусть $p_{j}$ - вероятность исхода $E_{j}$ в каждом испытании, $j=0,1, \ldots, N, p_{0}+p_{1}+\ldots+p_{N}=1$. Будем предполагать, что положительные вероятности $p_{0}, p_{1}, \ldots, p_{N}$ фиксированы. Если испытания проводятся до $r$-й реализации исхода $E_{0}, r=1,2, \ldots$ и $\eta(r)=\left(\eta_{1}(r), \ldots, \eta_{N}(r)\right)$, где $\eta_{j}(r)$ - число реализаций исхода $E_{j}$ в момент остановки испытаний, $j=1, \ldots, N$, TO

$$
\mathbf{P}\{\eta(r)=k\}=\frac{\left(k_{1}+\ldots+k_{N}+r-1\right) !}{(r-1) ! k_{1} ! \ldots k_{N} !} p_{0}^{r} \prod_{j=1}^{N} p_{j}^{k_{j}}
$$

где $k=\left(k_{1}, \ldots, k_{N}\right)$ и $k_{1}, \ldots, k_{N}$ - целые неотрицательные числа.

Формула (1) определяет так называемое отрицательное полиномиальное распределение случайного вектора $\eta(r)$. Это распределение можно рассматривать как многомерный аналог отрицательного биномиального распределения, получаемого из (1) при $N=1$. Подробную информацию об отрицательном полиномиальном распределении (включая и его асимптотический анализ при $r \rightarrow \infty$ ) можно найти в [1]. Из 
результатов, изложенньх в [1], в частности, следует; что

$$
\begin{aligned}
\mathbf{E} \eta_{j}(r) & =\frac{r p_{j}}{p_{0}}, \quad j=1, \ldots, N, \\
\mathbf{E}\left(\prod_{j=1}^{N} z_{j}^{\eta_{j}(r)}\right) & =p_{0}^{r}\left(f\left(z_{1}, \ldots, z_{N}\right)\right)^{r},
\end{aligned}
$$

где

$$
f\left(z_{1}, \ldots, z_{N}\right)=\left(1-\sum_{j=1}^{N} p_{j} z_{j}\right)^{-1}
$$

Из равенств (3) и (4) получаем, что в обозначениях (1)

$$
\begin{aligned}
\mathbf{P}\{\eta(r)=k\} & =\mathbf{P}\left\{\eta_{j}(r)=k_{j} ; j=1, \ldots, N\right\} \\
& =\frac{p_{0}^{r}}{(2 \pi i)^{N}} \oint \ldots \oint \frac{\left(f\left(z_{1}, \ldots, z_{N}\right)\right)^{r}}{z_{1}^{k_{1}+1} \ldots z_{N}^{k_{N}+1}} d z_{1} \ldots d z_{N}
\end{aligned}
$$

где интегрирование осуществляется по пути $A_{1} \times \ldots \times A_{N}$ в $\mathbf{C}^{N}$ и $A_{j}$ - окружность $\left|z_{j}\right|=R_{j}>0$ с центром в нуле, пробегаемая в положительном направлении, $j=1, \ldots, N$, причем

$$
\sum_{j=1}^{N} p_{j} R_{j}<1
$$

В статье, исходя из интегрального представления (5), найдены асимптотические оценки вероятностей больших уклонений

$$
\begin{aligned}
& \mathbf{P}\left\{\eta_{j}(r) \geqslant k_{j}, j=1, \ldots, N\right\}, \\
& \mathbf{P}\left\{\eta_{j}(r) \leqslant k_{j}, j=1, \ldots, N\right\}
\end{aligned}
$$

в предположении, что $r \rightarrow \infty$ and $k_{1}, \ldots, k_{N} \rightarrow \infty$ так, что

$$
\beta_{j}=\frac{k_{j}}{r} \geqslant \varepsilon>0, \quad j=1, \ldots, N
$$

где $\varepsilon$ - постоянная, а также при некоторых дополнительных условиях на параметры $\beta_{1}, \ldots, \beta_{N}$. При этом используется многомерный метод перевала в варианте И. Гуда (см. [2], с. 136-137]. В идейном плане изложение следует статье автора [3], где получены аналогичные оценки вероятностей больших уклонений для полиномиального распределения (хотя в рассматриваемой ситуации соответствующие результаты формулируются несколько сложнее).

Лемма 1. Пусть $r \rightarrow \infty u k_{1}, \ldots, k_{N} \rightarrow \infty \operatorname{max,~что~справедливо~условие~(7).~}$ Тогда равномерно относительно $\beta_{1}, \ldots, \beta_{N}$

$$
\begin{aligned}
\mathbf{P}\{\eta(r)=k\}=\frac{1}{\sqrt{\beta_{1} \ldots \beta_{N}(1+\beta)}} & (2 \pi r)^{-N / 2} \\
& \quad \times(1+\beta)^{r(1+\beta)} \exp \left(-r I\left(\beta_{1}, \ldots, \beta_{N}\right)\right)(1+O(1 / r)),
\end{aligned}
$$


əдe

$$
\begin{aligned}
I\left(\beta_{1}, \ldots, \beta_{N}\right) & =\sum_{j=1}^{N} \beta_{j} \ln \frac{\beta_{j}}{p_{j}}-\ln p_{0}>0 \\
\beta & =\sum_{j=1}^{N} \beta_{j} .
\end{aligned}
$$

Доказательство. Оценка (8) обосновывается с помощью формулы Стирлинга, если подставить в (1) значения $k_{j}=r \beta_{j}, j=1, \ldots, N$, и использовать (7), (9) и (10). Лемма 1 доказана.

В частности, если при условиях леммы 1 все числа $p_{j} / p_{0}=E \eta_{j}(r) / r$ (см. (2)) рациональны и $\beta_{j}=p_{j} / p_{0}, j=1, \ldots, N$, то из (8) получаем, что

$$
\mathbf{P}\{\eta(r)=k\}=\frac{p_{0}^{(N+1) / 2}}{\sqrt{p_{1} \ldots p_{N}}}(2 \pi r)^{-N / 2}(1+O(1 / r)) .
$$

Последнее равенство является частным случаем локальной нормальной теоремы для случайного вектора $\eta(r)=\left(\eta_{1}(r), \ldots, \eta_{N}(r)\right)$ (общий случай см. в [1])

Лемма 2. Если $k_{1}, \ldots, k_{N}$ - целъе неотрицателънъе числа, то

$$
\begin{aligned}
\mathbf{P}\left\{\eta_{j}(r) \geqslant k_{j}, j=\right. & 1, \ldots, N\} \\
& =\frac{p_{0}^{r}}{(2 \pi i)^{N}} \oint \ldots \oint \frac{\left(f\left(z_{1}, \ldots, z_{N}\right)\right)^{r}}{z_{1}^{k_{1}+1} \ldots z_{N}^{k_{N}+1}} \prod_{j=1}^{N}\left(1-z_{j}^{-1}\right)^{-1} d z_{1} \ldots z_{N},
\end{aligned}
$$

где функция $f\left(z_{1}, \ldots, z_{N}\right)$ определяется согласно (4) и интегрирование осуществляется по тому же пути, что и в (5), для которого выполнено условие (6) $u$ неравенства

$$
R_{j}>1, \quad j=1, \ldots, N
$$

Доказателъство. При выполнении условий леммы 2 и (5)

$$
\begin{aligned}
\mathbf{P}\left\{\eta_{j}(r) \geqslant k_{j}, j=1, \ldots, N\right\}=\sum_{l_{1}=k_{1}}^{\infty} \ldots \sum_{l_{N}}^{\infty} \mathbf{P}\left\{\eta_{j}(r)=l_{j}, j=1, \ldots, N\right\} \\
=\frac{p_{0}^{r}}{(2 \pi i)^{N}} \oint \ldots \oint \frac{\left(f\left(z_{1}, \ldots, z_{N}\right)\right)^{r}}{z_{1} \ldots z_{N}} \sum_{l_{1}=k_{1}}^{\infty} z_{1}^{-l_{1}} \ldots \sum_{l_{N}=k_{N}}^{\infty} z_{N}^{-l_{N}} d z_{1} \ldots d z_{N} .
\end{aligned}
$$

Суммируя геометрические прогрессии под знаком многомерного интеграла и учитывая (12), получаем (11). Лемма доказана.

Теорема 1. Пусть $r \rightarrow \infty u k_{1}, \ldots, k_{N} \rightarrow \infty$ тах, ито $\beta_{j}=k_{j} / r-$ фиксированнъе положительные числа, для которых

$$
\beta_{j} /(1+\beta)>p_{j}, \quad j=1, \ldots, N .
$$


Tогдa

$$
\mathbf{P}\left\{\eta_{j}(r) \geqslant k_{j}, j=1, \ldots, N\right\}=\mathbf{P}\{\eta(r)=k\} \prod_{j=1}^{N}\left(1-p_{j}(1+\beta) / \beta_{j}\right)^{-1}(1+O(1 / r)),
$$

где вероятность $\mathbf{P}\{\eta(r)=k\}$ и величина $\beta$ определяются согласно (8), (9), (10).

Доказательство. Заметим, что при условиях теоремы 1 выполняются неравенства (7), поэтому согласно лемме 1 справедливо равенство (8). Чтобы получить из (8) оценку (14), используем интегральное представление (11). Для оценки интеграла в (11) применим многомерный метод перевала. Точки перевала подынтегральной функции (без учета фигурирующего в выражении этой функции произведения) определяются из системы уравнений

$$
r z_{j} \frac{\partial d}{\partial d z_{j}} f\left(z_{1}, \ldots, z_{N}\right)=k_{j} f\left(z_{1}, \ldots, z_{N}\right), \quad j=1, \ldots, N .
$$

Подставляя значение $f\left(z_{1}, \ldots, z_{N}\right)$ из (4) и $k_{j}=r \beta_{j}, j=1, \ldots, N$, получаем эквивалентную систему

$$
p_{j} z_{j}=\beta_{j}\left(1-\sum_{i=1}^{N} p_{i} z_{i}\right), \quad j=1, \ldots, N
$$

Решение системы (15) будем искать в виде $z_{j}=R_{j}>0, j=1, \ldots, N$. Такое решение определяется равенствами

$$
R_{j}=\frac{\beta_{j}}{p_{j}(1+\beta)}, \quad j=1, \ldots, N
$$

При условиях теоремы 1 из (10) следует, что $\beta>0$, поэтому $p_{1} R_{1}+\ldots+p_{N} R_{N}=$ $\beta /(1+\beta)<1$, то есть выполнено условие (6). Кроме того, из (13) следует (12). Таким образом, при условиях теоремы 1 справедливо интегральное представление (11). При этом

$$
f\left(R_{1}, \ldots, R_{N}\right)=1+\beta
$$

Согласно [2], с учетом (16) справедливы равенства

$$
\begin{aligned}
& \mathbf{P}\left\{\eta_{j}(r) \geqslant k_{j}, j=1, \ldots, N\right\} \\
& =p_{0}^{r} \prod_{j=1}^{N}\left(1-p_{j}(1+\beta) / \beta_{j}\right)^{-1} \frac{\left(f\left(R_{1}, \ldots, R_{N}\right)\right)^{r}}{(2 \pi r)^{N / 2} R_{1}^{k_{1}} \ldots R_{N}^{k_{N}} \sqrt{\Delta}}(1+O(1 / r)) \\
& =\prod_{j=1}^{N}\left(1-p_{j}(1+\beta) / \beta_{l}\right)^{-1}(2 \pi r)^{-N / 2}(1+\beta)^{r(1+\beta)} \\
& \quad \times \exp \left(-r I\left(\beta_{1}, \ldots, \beta_{N}\right)\right) \Delta^{-1 / 2}(1+O(1 / r)),
\end{aligned}
$$

где величина $I\left(\beta_{1}, \ldots, \beta_{N}\right)$ определяется в (9) и $\Delta=\operatorname{det}\left(\Delta_{i j}\right), i, j=1, \ldots, N$, причем при $i \neq j$

$$
\Delta_{i j}=z_{i} \frac{\partial d}{\partial d z_{i}}\left(z_{j} \frac{\partial d}{\partial d z_{j}}(\ln f)\right)\left(R_{1}, \ldots, R_{N}\right)=\beta_{i} \beta_{j}
$$


и при $i=j$

$$
\Delta_{i i}=z_{i} \frac{\partial d}{\partial d z_{i}}\left(z_{i} \frac{\partial d}{\partial d z_{i}}(\ln f)\right)\left(R_{1}, \ldots, R_{N}\right)=\beta_{i}\left(1+\beta_{i}\right) .
$$

Следовательно,

$$
\Delta=\beta_{1} \ldots \beta_{N}(1+\beta)>0 .
$$

Из оценок (8), (17) и равенства (18) получаем (14). Теорема 1 доказана.

Замечание 1. Из условий (13), в частности, следует, что

$$
\frac{\beta}{1+\beta}>\sum_{j=1}^{N} p_{j}=1-p_{0}
$$

то есть $1+\beta>1 / p_{0}$. Поэтому

$$
\beta_{j}>p_{j}(1+\beta)>p_{j} / p_{0}=\frac{1}{r} \mathrm{E} \eta_{j}(r), \quad j=1, \ldots, N .
$$

Таким образом, из (13) следуют неравенства (19). При $N=1$ верно и обратное, однако при $N \geqslant 2$ это уже не так.

Замечание 2. Множество $E$ всех $N$-мерных векторов $\left(\beta_{1}, \ldots, \beta_{N}\right)$ с положительными координатами, удовлетворяющих условиям (13), можно представить в виде

$$
E=\bigcup_{c>\left(1-p_{0}\right) / p_{0}} E(c)
$$

где при $c>\left(1-p_{0}\right) / p_{0}$

$$
E(c)=\left\{\left(\beta_{1}, \ldots, \beta_{N}\right): \beta_{1}+\ldots+\beta_{N}=c, \beta_{j}>(1+c) p_{j}, j=1, \ldots, N\right\} \neq \varnothing
$$

так как

$$
\sum_{j=1}^{N}(1+c) p_{j}=(1+c)\left(1-p_{0}\right)<c .
$$

Замечание 3. Утверждение теоремы 1 останется справедливым при отказе от условия, что $\beta, j=1, \ldots, N$, фиксированы, если неравенства (13) усилить, предположив, что

$$
\left(\beta_{1}, \ldots, \beta_{N}\right) \in E^{*},
$$

где $E^{*}$ - произвольное компактное подмножество $E$. При этом оценка (14) будет выполняться равномерно относительно $\left(\beta_{1}, \ldots, \beta_{N}\right) \in E^{*}$.

Лемма 3. Если $k_{1}, \ldots, k_{N}$ - челье неотрицателъные числа, то

$$
\begin{aligned}
\mathbf{P}\left\{\eta_{j}(r) \leqslant k_{j}, j=1, \ldots, N\right\}=\frac{p_{0}^{r}}{(2 \pi i)^{N}} & \oint \ldots \oint \frac{\left(f\left(z_{1}, \ldots, z_{N}\right)\right)^{r}}{z_{1}^{k_{1}+1} \ldots z_{N}^{k_{N}+1}} \\
& \times \prod_{j=1}^{N}\left(\left(1-z_{j}\right)^{-1}\left(1-z_{j}^{k_{j}+1}\right)\right) d z_{1} \ldots d z_{N}
\end{aligned}
$$

где интегрирование осущеспвляется по тому же пути, что и в (5), и выполнены условия (6) и неравенства $R_{j} \neq 1, j=1, \ldots, N$. 
Доказательство. Равенство (20) следует из (5) с учетом соотношений

$$
\mathbf{P}\left\{\eta_{j}(r) \leqslant k_{j}, j=1, \ldots, N\right\}=\sum_{l_{1}=0}^{k_{1}} \ldots \sum_{l_{N}=0}^{k_{N}} \mathbf{P}\left\{\eta_{j}(r)=l_{j}, j=1, \ldots, N\right\}
$$

если просуммировать геометрические прогрессии под знаком многомерного интеграла. Лемма 3 доказана.

Теорема 2. Пусть $r \rightarrow \infty u k_{1}, \ldots, k_{N} \rightarrow \infty$ max, что $\beta_{j}=k_{j} / r-$ фиксированнъе положительные числа, для которых

$$
\beta_{j} /(1+\beta)<p_{j}, \quad j=1, \ldots, N
$$

Tогда

$$
\mathbf{P}\left\{\eta_{j}(r) \leqslant k_{j}, j=1, \ldots, N\right\}=\mathbf{P}\{\eta(r)\} \prod_{j=1}^{N}\left(1-\frac{\beta_{j}}{p_{j}(1+\beta)}\right)^{-1}(1+O(1 / r)),
$$

где вероятность $\mathbf{P}\{\eta(r)=k\}$ и величина $\beta$ определяются согласно (8), (9), (10).

Доказательство. При условиях теоремы 2 оценка (8) по-прежнему выполняется, поэтому достаточно оценить многомерный интеграл (20), используя неравенства (21). Из этих керавенств получаем, что

$$
\left|z_{j}\right|=R_{j}<1, \quad j=1, \ldots, N
$$

и поэтому

$$
\prod_{j=1}^{N}\left(1-z_{j}^{k_{j}+1}\right)=1+O(1 / r)
$$

равномерно относительно $z_{1}, \ldots, z_{N}$.

Следовательно, с учетом (20) можно утверждать, что

$$
\begin{aligned}
& \mathbf{P}\left\{\eta_{j}(r) \leqslant k_{j}, j=1, \ldots, N\right\} \\
& \quad=\frac{p_{0}^{r}}{(2 \pi i)^{N}} \oint \ldots \oint \frac{\left(f\left(z_{1}, \ldots, z_{N}\right)\right)^{r}}{z_{1}^{k_{1}+1} \ldots z_{N}^{k_{N}}+1} \prod_{j=1}^{N}\left(1-z_{j}\right)^{-1} d z_{1} \ldots d z_{N}(1+O(1 / r)) .
\end{aligned}
$$

Применяя к оценке интеграла (23) многомерный метод перевала и проводя рассуждения, аналогичные использованным при доказательстве теоремы 1 , получаем (22). Теорема 2 доказана.

Замечание 4. Из условий (21), в частности, следует, что $1+\beta<1 / p_{0}$, и поэтому

$$
\beta_{j}<p_{j}(1+\beta)<p_{j} / p_{0}=\frac{1}{r} \mathrm{E} \eta_{j}(r), \quad j=1, \ldots, N .
$$

Таким образом, из (21) следуют неравенства (24). При $N=1$ верно и обратное, однако при $N \geqslant 2$ это не так. 
Замечание 5. Множество $F$ всех $N$-мерных векторов $\left(\beta_{1}, \ldots, \beta_{N}\right)$ с. положительными координатами, удовлетворяющих условиям (21), может быть представлено в виде

$$
F=\bigcup_{0<c<\left(1-p_{0}\right) / p_{0}} F(c),
$$

где при $c \in\left(0,\left(1-p_{0}\right) / p_{0}\right)$

$$
F(c)=\left\{\left(\beta_{1}, \ldots, \beta_{N}\right): \beta_{1}+\ldots \beta_{N}=c, \beta_{j}<(1+c) p_{j}, j=1, \ldots, N\right\} \neq \varnothing,
$$

поскольку при таких значениях $c$

$$
\sum_{j=1}^{N}(1+c) p_{j}=(1+c)\left(1-p_{0}\right)>c .
$$

Замечание 6. Утверждение теоремы 2 остается справедливым и при отказе от условия, что $\beta_{j}, j=1, \ldots, N$, фиксированы, если неравенства (21) усилить, предположив, что

$$
\left(\beta_{1}, \ldots, \beta_{N}\right) \in F^{*},
$$

где $F^{*}$ - произвольное компактное подмножество $F$. При этом оценка (22) будет выполняться равномерно относительно $\left(\beta_{1}, \ldots, \beta_{N}\right) \in F^{*}$.

\section{Список литературы}

1. Ивченко Г. И., Морозова Н. М., Отрицательное полиномиальное распределение. Дискретная математика (1993) 5, №2, 138-149.

2. Егорычев Г. П., Интегралъное представление и вычисление комбинаторных сумм. Наука, Новосибирск, 1977.

3. Тимашев А. Н., Теоремы о больших уклонениях в полиномиальной схеме испытаний. Дискретная математика (1993) 5, №2, 83-89.

Статья поступила 25.05.2000. 